Published in Kennedy J., de Queiroz R. (eds) Logic, Language, Information, and Computation. WoLLIC 2017. Lecture Notes in Computer Science, vol 10388. Berlin: Springer. ISBN:978-3-662-55386-2.

https://doi.org/10.1007/978-3-662-55386-2_2

\title{
Independence-Friendly logic without Henkin quantification
}

\author{
Fausto Barbero ${ }^{1}$, Lauri Hella ${ }^{2}$, and Raine Rönnholm ${ }^{2}$ \\ 1 University of Helsinki, Philosophy, Faculty of Arts, Finland \\ fausto.barbero@helsinki.fi \\ 2 University of Tampere, Mathematics, Faculty of Natural Sciences, Finland \\ \{lauri.hella, raine.ronnholm\}@uta.fi
}

\begin{abstract}
We analyze from a global point of view the expressive resources of IF logic that do not stem from Henkin (partially-ordered) quantification. When one restricts attention to regular IF sentences, this amounts to the study of the fragment of IF logic which is individuated by the game-theoretical property of Action Recall. We prove that the fragment of Action Recall can express all existential second-order (ESO) properties. This can be accomplished already by the prenex fragment of Action Recall, whose only second-order source of expressiveness are the so-called signalling patterns. The proof shows that a complete set of Henkin prefixes is explicitly definable in the fragment of Action Recall. In the more general case, in which also irregular IF sentences are allowed, we show that full ESO expressive power can be achieved using neither Henkin nor signalling patterns.
\end{abstract}

\section{Introduction}

Independence-Friendly logic $([10],[15])$ is one of a number of formalisms that have been developed in order to make various notions of dependence and independence accessible to the instruments of logical investigation. IndependenceFriendly (IF) logic and similar formalisms (Dependence-Friendly logic, Dependence logic [18]), in particular, were developed as a more flexible approach to the logic of Henkin quantifiers ([9]). The Henkin quantifier $\mathrm{H}_{k}^{n}$ is a matrix

$$
\left(\begin{array}{ccccc}
\forall x_{1}^{1} & \forall x_{1}^{2} & \ldots & \forall x_{1}^{n} & \exists y_{1} \\
\vdots & \vdots & \ddots & \vdots & \vdots \\
\forall x_{k}^{1} & \forall x_{k}^{2} & \ldots & \forall x_{k}^{n} & \exists y_{k}
\end{array}\right)
$$

which, differently from a linear sequence of the same quantifiers, is meant to state that each $y_{i}$ is supposed to be chosen as a function of $x_{i}^{1}, \ldots, x_{i}^{n}$ only. In IF logic, the same is achieved by means of a linear prefix, together with a slashing device. For example, the Henkin quantifier $\mathrm{H}_{2}^{1}$ is expressed in IF logic by the sequence of quantifiers

$$
\forall x_{1}^{1} \exists y_{1} \forall x_{2}^{1}\left(\exists y_{2} /\left\{x_{1}^{1}, y_{1}\right\}\right) .
$$

The slashed quantifier $\left(\exists y_{2} /\left\{x_{1}^{1}, y_{1}\right\}\right)$ expresses the fact that $y_{2}$ is independent from $x_{1}^{1}$ and $y_{1}$. 
It has been gradually realized that, in spite of the fact that it stems from the study of Henkin quantifiers, IF logic derives its expressiveness also from other sources. Henkin quantifiers are partial orderings of first-order quantifiers; but in IF logic also intransitive (thus not ordered) dependence sequences are allowed, for example

$$
\forall x \exists y(\exists z /\{x\}) .
$$

Here $y$ depends on $x, z$ depends on $y$, but $z$ does not depend on $x$. It is known that such quantifier sequences, also known as signalling sequences (or patterns) can be used to express higher-order concepts ([7],[4],[17]); for example, the IF sentence $\exists v \forall x \exists y(\exists z /\{x\})(x=z \wedge y \neq v)$ is known to characterize the class of all infinite structures (this idea is attributed, in [7], to Fred Galvin).

Henkin and signalling patterns are known to exhaust the higher-order expressive power of prenex regular ${ }^{3}$ IF logic: if a regular sentence is in prenex normal form and does not contain Henkin or signalling patterns, then it is equivalent to some first-order sentence ([17]). Non-prenex, regular IF logic is known to contain further expressive synctactical patterns (involving the interaction of quantifiers and disjunctions) that are neither of the Henkin nor the signalling type, yet allow describing NP-complete problems such as SAT and SET PARTITIONING ([2]); the problem of a complete classification of such patterns is still open. Less is known of irregular IF logic, which will be addressed here in Section 5. The aim of the present paper is a better understanding of the resources of IF logic that do not stem from Henkin quantification.

A peculiarity of Independence-Friendly logic is the close link between its syntax and the theory of extensive games of imperfect information. The link is given by the so-called Game-Theoretical Semantics, that we will review in Section 2. Through this connection, game-theoretical concepts throw light on peculiarities of the logic; and vice versa, the study of logical phenomena can cast new light on the foundations of game theory.

It is well known, through the works of Henkin, Hintikka and others, that it is possible to define a notion of truth for first-order languages in terms of certain games of perfect information, which involve two players called Verifier ("Eloise") and Falsifier ("Abelard"), who take it in turns to point out evidence for or against the truth of a given sentence $\varphi$ in a given structure $M$. The resulting GameTheoretical Semantics (GTS) is equivalent to the usual Tarskian one.

When moving from first-order to IF languages, extending the Tarskian semantics is not straightforward ${ }^{4}$; instead, it is quite natural to generalize the semantic games by allowing imperfect information, in a way that the independence constraints expressed by syntax correspond (roughly speaking) to the fact that a player is forced to make his/her choices in ignorance of the outcomes of some earlier moves ([10]). This generalization allows new complex possibilities. Many IF games are actually games of imperfect recall: the players may forget what they knew at earlier stages of the game.

\footnotetext{
3 The notion of regularity will be defined in Section 2 .

${ }^{4}$ It can be done, at the cost of defining a notion of satisfaction by sets of assignment, instead of the usual single assignments. See e.g. [11],[12],[5],[18],[15].
} 
In this paper, we will be particularly interested in a game-theoretical property called action recall. Eloise has action recall if she cannot forget her own moves; assuming regularity, an IF sentence has action recall (i.e., all its corresponding games have action recall) for Eloise if its sets of slashed variables associated to existential quantifiers contain no existentially quantified variables. Thus for example $\forall x(\exists y /\{x\}) R(x, y)$ has action recall, while $\exists x(\exists y /\{x\}) R(x, y)$ does not.

The fragment of sentences with action recall for Eloise is particularly important, because, in it, it is impossible to write the usual IF translations of Henkin prefixes ${ }^{5}$, and yet, it is a highly expressive fragment. Therefore, it is natural to wonder to what degree the IF-definable concepts are expressible under the restriction of action recall. IF logic is known to capture exactly existential second-order (ESO) definable classes. In Section 4 we will show that the Henkin prefixes $\mathrm{H}_{2}^{n}$ are explicitly definable in the prenex, regular fragment of action recall (therefore, by means of signalling). The $\mathrm{H}_{2}^{n}$ prefixes, taken together, are known to capture all ESO definable concepts ([14]); therefore, the prenex, regular fragment of action recall suffices for full IF expressive power.

When irregular IF sentences are allowed, instead, it becomes possible to violate action recall in new ways; in Section 5 we use this fact to show, by means of a translation procedure, that it is possible to express all IF properties using neither Henkin nor signalling quantifier patterns. Section 2 reviews preliminary notions about game-theoretical semantics and action recall, while Section 3 presents some significant examples.

\section{Preliminaries}

Notation. Structures are denoted by capital italic letters. To keep the notation simple, we do not introduce a separate symbol for the domain of a structure; thus if $M$ is a structure, $a \in M$ and $R \subseteq M^{2}$ mean that $a$ is an element of the domain of $M$ and $R$ is a binary relation on the domain of $M$, respectively.

An assignment of variables on a structure $M$ is a function $s: V \rightarrow M$, where the domain $V$ of $s$ is a finite set of variables. We denote the set of all assignments on $M$ with domain $V$ by $\operatorname{As}(V, M)$. Given an assignment $s \in \operatorname{As}(V, M)$ and an element $a \in M$, we write $s(a / v)$ for the assignment with domain $V \cup\{v\}$ such that $s(a / v)(v)=a$ and $s(a / v)(u)=s(u)$ for $u \in V \backslash\{v\}$. If $\bar{x}=\left(x_{1}, \ldots, x_{n}\right)$ is a tuple of variables, we use the shorthand notation $s(\bar{x})$ for $\left(s\left(x_{1}\right), \ldots, s\left(x_{n}\right)\right)$.

Game-Theoretical Semantics. The syntax of IF logic is a restriction of the usual first-order syntax, to which we add quantifiers of the forms $(\exists v / V)$ and $(\forall v / V)$, where $V$ is a finite set of variables, called the slash set of the quantifier. When $V=\emptyset$, we use the abbreviation $Q x:=(Q x / V)$. The syntax is restricted, with regards to usual first-order languages, in that

- we only allow the connectives $\wedge, \vee$ and $\neg$, and

- for simplicity, we only allow $\neg$ to occur in front of atomic formulae.

\footnotetext{
${ }^{5}$ This point is exemplified by the IF rendition of the $\mathrm{H}_{2}^{1}$ prefix, shown above: its "slash set" $\left\{x_{1}^{1}, y_{1}\right\}$ contains an existentially quantified variable, $y_{1}$.
} 
The set Free $(\varphi)$ of free variables of a formula $\varphi$ is defined as usual, with the proviso that also variables from slash sets can be either free or bound. For example, in $\forall x(\exists y /\{x, y, z\}) \psi$, the occurrence of $x$ in the slash set is bound, while the occurrences of $y, z$ are free.

A further restriction on the syntax of IF logic that is often assumed in the literature (see, e.g., [3] and [15]) is that variables are not requantified:

- A sentence is regular if no quantifier $(Q v / V)$ occurs in the scope of another quantifier $\left(Q^{\prime} v / W\right)$ over the same variable $v$.

We denote IF logic with this regularity restriction by $\mathrm{IF}^{\mathrm{r}}$. We will mostly restrict our studies to $\mathrm{IF}^{\mathrm{r}}$, but in Section 5 we will also consider irregular sentences.

Game-Theoretical Semantics (GTS) associates to each triple $(\varphi, M, s)$, where $\varphi$ is an IF formula, $M$ is a structure, and $s \in \operatorname{As}(V, M)$ for a set $V$ of variables such that $\operatorname{Free}(\varphi) \subseteq V$, a 2-player win-lose extensive game of imperfect information $G(\varphi, M, s)$. In case $\varphi$ is a sentence and $s=\emptyset$, we simply write $G(\varphi, M)$. The two players, usually called Eloise and Abelard, can be thought of as trying to verify, respectively falsify, the sentence $\varphi$ on the structure $M$. Their moves are triggered by the most external logical operator of $\varphi$ :

- in $G\left(\psi_{1} \vee \psi_{2}, M, s\right)$, Eloise chooses a disjunct $\psi_{i}$, and then $G\left(\psi_{i}, M, s\right)$ is played;

- in $G\left(\psi_{1} \wedge \psi_{2}, M, s\right)$, the same kind of move is performed by Abelard;

- in $G((\exists v / V) \psi, M, s)$, Eloise picks an element $a \in M$ and then the game $G(\psi, M, s(a / v))$ is played;

- in $G((\forall v / V) \psi, M, s)$ the same kind of move is performed by Abelard;

- in $G(\alpha, M, s)$, with $\alpha$ a literal (i.e., an atomic formula or the negation of an atomic formula), the winner is decided: it is Eloise in case $M, s \models \alpha$ (in the usual first-order sense), and Abelard otherwise.

Imperfect information manifests itself in that some histories of the game are considered indistinguishable for the player who has the turn to move at the end of them. If two histories $h$ and $h^{\prime}$ both end with the choice of a subgame associated with the same occurrence of a subformula $(Q v / V) \psi$ with assignments $s_{h}, s_{h^{\prime}} \in \operatorname{As}(W, M)$ such that $s_{h}(w)=s_{h^{\prime}}(w)$ for every $w \in W \backslash V$, then $h$ and $h^{\prime}$ are indistinguishable for the player associated to $(Q v / V)$, and we write $h \sim_{V} h^{\prime}$.

A strategy for Eloise in game $G(\varphi, M, s)$ is a function associating, to each history ending in a subgame $G\left((\exists v / V) \psi, M, s^{\prime}\right)$, an element $a \in M$; and, to every history ending in a subgame $G\left(\psi_{1} \vee \psi_{2}, M, s^{\prime}\right)$, either $\psi_{1}$ or $\psi_{2}$. Strategies for Abelard can be similarly defined.

A strategy of Eloise is winning if, playing according to it, Eloise wins, whatever moves Abelard makes. Winning strategies for Abelard are defined dually.

A strategy $\sigma$ is uniform if, whenever two histories $h, h^{\prime}$ are in its domain and $h \sim_{V} h^{\prime}$ (for the only appropriate $V$ ), then $\sigma(h)=\sigma\left(h^{\prime}\right)$.

With this game-theoretical apparatus, it is possible to define the notions of truth and falsity for IF sentences as the existence of appropriate strategies:

$M \models \varphi$ if Eloise has a uniform winning strategy in $G(\varphi, M)$

$M=^{-} \varphi$ if Abelard has a uniform winning strategy in $G(\varphi, M)$. 
There is also a third possibility: it may happen that neither player has a uniform winning strategy (consider, e.g., the sentence $\forall x(\exists y /\{x\}) x=y$ ). In that case, the game and the truth value of the sentence on $M$ are said to be undetermined. In this paper, we only focus on the truth/nontruth distinction. Accordingly, we say that a class $K$ of structures is definable in IF, if there is an IF sentence $\varphi$ such that for all structures $M$, we have $M \in K \Leftrightarrow M \models \varphi$. As was already shown in [10], IF logic has the same expressive power as existential second-order logic ESO: a class of structures is definable in IF if and only if it is definable in ESO.

Signalling and Henkin patterns. We have been talking informally of Henkin and signalling patterns of quantifiers. Exact definitions were given in [17]; We extend these definitions for irregular sentences.

- Let $(Q v / V)$ and $\left(Q^{\prime} u / U\right)$ be quantifiers occurring in a prefix or in a sentence. We use the following terminology:

- $(Q v / V)$ is in the effective scope of $\left(Q^{\prime} u / U\right)$, if $(Q v / V)$ is in the scope of $\left(Q^{\prime} u / U\right), v \neq u$, and there is no quantifier $\left(Q^{\prime \prime} u / W\right)$ in the scope of $\left(Q^{\prime} u / U\right)$ such that $(Q v / V)$ in the scope of $\left(Q^{\prime \prime} u / W\right)$. We write $(Q v / V) \in \operatorname{Es}\left(Q^{\prime} u / U\right)$ if this is the case.

- $(Q v / V)$ depends on $\left(Q^{\prime} u / U\right)$ if $(Q v / V) \in \operatorname{Es}\left(Q^{\prime} u / U\right)$ and $u \notin V$.

- A signalling pattern in a sentence consists of three quantifiers $(\forall x / X),(\exists y / Y)$, $(\exists z / Z)$ such that $(\exists z / Z) \in \operatorname{Es}(\exists y / Y) \cap \operatorname{Es}(\forall x / X),(\exists y / Y) \in \operatorname{Es}(\forall x / X)$ and

- $(\exists y / Y)$ depends on $(\forall x / X)$;

- $(\exists z / Z)$ depends on $(\exists y / Y)$, but not on $(\forall x / X)$.

- A Henkin pattern in a sentence consists of four quantifiers $(\forall x / X),(\exists y / Y)$, $(\forall z / Z),(\exists w / W)$ such that we have $(\exists w / W),(\forall z / Z),(\exists y / Y) \in \operatorname{Es}(\forall x / X)$, $(\exists w / W) \in \operatorname{Es}(\exists y / Y) \cap \operatorname{Es}(\forall z / Z)$ and

- $(\exists y / Y)$ depends on $(\forall x / X)$, but not on $(\forall z / Z)$;

- $(\exists w / W)$ depends on $(\forall z / Z)$, but not on $(\forall x / X)$ or $(\exists y / Y)$.

Note that the last condition holds only if the existentially quantified variable $y$ is in the slash set $W$. Also note that in the case of regular sentences, we may simply talk about scopes instead of effective scopes in the definitions above.

Action recall fragment of IF logic. Even though many different games are associated to each single sentence (one game for each sentence-structure pair), some interesting properties of the games are characterized by synctactical properties of the associated sentences; they are invariants of the sentence alone. As a consequence, such game-theoretical properties define associated fragments of IF logic. In particular, in the literature $([13],[16],[15]$ sect. $6.4,[1])$ there has been some interest in properties that limit the ability of players to forget. Considering for example the role of Eloise:

- Eloise has action recall if she cannot forget her own moves.

- Eloise has knowledge memory if she cannot forget what she knew at earlier stages of the game.

- Eloise has perfect recall if she has both action recall and knowledge memory. 
Under the assumption of regularity, each of these properties has been given a syntactical characterization in the literature (see e.g., [15], [1]). For action recall the characterization is as follows: Assume that $\varphi$ is a regular IF sentence. Then Eloise has action recall in the game $G(\varphi, M, s)$ if and only if $\varphi$ satisfies the following restriction on slash sets:

(RS) If an existential quantifier $(\exists v / V)$ occurs in the scope of another existential quantifier $(\exists u / U)$, then $u \notin V$.

It should be noted that the condition (RS) does not guarantee action recall for Eloise on irregular sentences. For example, the formula $\exists x \exists x \exists y(x=y)$ violates action recall: in her third move, Eloise forgets the value chosen in the first move, because it has been overwritten by the second move.

We denote the fragment of IF consisting of all regular formulae that satisfy (RS) by $\operatorname{IF}_{\mathrm{AR}(\exists)}^{\mathrm{r}}$ (here $\operatorname{AR}(\exists)$ stands for Action Recall for $\exists$ loise). Note that it is impossible to write a Henkin quantifier in $\mathrm{IF}_{\mathrm{AR}(\exists)}^{\mathrm{r}}$; for this reason, the fragment $\mathrm{IF}_{\mathrm{AR}(\exists)}^{\mathrm{r}}$ will be one of the main objects of study in this paper. We will also be interested in the set $\mathrm{IF}_{\mathrm{AR}(\exists)}^{\mathrm{p}, \mathrm{r}}$ of prenex sentences of $\operatorname{IF}_{\mathrm{AR}(\exists)}^{\mathrm{r}}$.

The fragments of perfect recall and knowledge memory are relatively wellunderstood; truth, in both of them, can only capture first-order concepts (for the former fragment, the result was anticipated in [13], [16] and adequately proved in [15]; the latter fragment was addressed in [1]). The regular action recall fragment $\mathrm{IF}_{\mathrm{AR}(\exists)}^{\mathrm{r}}$ is by far less understood; some examples in the literature show that it is capable of expressing higher-order concepts, such as infinity over the empty signature, and some NP-complete problems (see Section 3). But a general understanding of its expressive power is lacking, and will be addressed in the present paper.

\section{Examples}

The main result that will be proved in section 4 implies that any ESO concept can be expressed by some regular, prenex action recall formula (therefore, by means of signalling). However, the defining sentences provided by the theorem are often unnecessarily complicated. We give here some examples of NP-complete problems that can be expressed by relatively simple sentences of $\operatorname{IF}_{\mathrm{AR}(\exists)}^{\mathrm{p}, \mathrm{r}}$.

Example 1. In [17], it was shown that the EXACT COVER BY 3-SETS problem can be defined by a $\operatorname{IF}_{\mathrm{AR}(\exists)}^{\mathrm{p}, \mathrm{r}}$ sentence. This problem consists in deciding, given a set $U$ of $3 k$ elements and a family $C$ of 3 -element subsets of $U$, whether there is a subfamily of $C$ which is a partition of $U$. It is defined by the sentence

$$
\forall x \exists y(\exists z / x)(U(x) \rightarrow(K(y) \wedge E(x, z)))
$$

on finite structures $M$ of domain $U \cup C$ (where $U \cap C=\emptyset$ ), such that $U^{M}=U$, $\operatorname{Card}\left(K^{M}\right)=k$ and $E^{M}=\{\langle a, B\rangle \mid a \in U, B \in C, a \in B\}$. We wish to point out that, if we restrict, w.l.o.g., the class of structures by the additional constraint that $K^{M} \subseteq M \backslash U^{M}$, then the condition above can be shown (see Appendix) to be equivalent to an ESO sentence of prefix $\exists f \forall x$ :

$$
\varphi^{*}=\exists f \forall x(U(x) \rightarrow(K(f(x)) \wedge E(x, f(f(x))))) .
$$


$\exists f \forall x$ is the simplest non-trivial prefix of functional ESO. The fact that it can capture NP-complete problems was shown by Grandjean ([8]); he applied this prefix to a conjunction of twenty-one clauses to define the HAMILTON PATH problem.

Example 2. We consider another NP-complete problem, DOMINATING SET: the problem of deciding, given an integer $k$ and a graph $G=\left(V, E^{G}\right)$ as input, whether there is a set $D \subseteq V$ of vertices of size at most $k$ such that for every vertex $x \in V$ : either $x \in \bar{D}$ or $(y, x) \in E^{G}$ for some $y \in D$. Assuming that the intended structures encode $k$ by an interpreted unary predicate $P^{G}$ of cardinality $k$, the problem is described (see Appendix) by the $\operatorname{IF}_{\mathrm{AR}(\exists)}^{\mathrm{p}, \mathrm{r}}$ sentence

$$
\forall x \exists z(\exists y /\{x\})((E(y, x) \vee y=x) \wedge P(z)) .
$$

This description is based on an analogous result for Dependence logic ([19]).

Example 3. Also the problem SAT is expressible by means of signalling. SAT is stated as follows: given a propositional formula $\pi$ in conjunctive normal form, is $\pi$ satisfiable? The problem can be modeled over structures $M$ of signature $P, N, C, 0,1$, with $0^{M}, 1^{M}$ distinct constants; $C^{M} \subseteq M$ representing the set of clauses; $P^{M}, N^{M} \subseteq\left(M \backslash C^{M}\right) \times C^{M}$, representing the fact that the first argument occurs positively, respectively negatively, in the second argument. In this class of structures, SAT is described (see Appendix) by the following $\mathrm{IF}_{\mathrm{AR}(\exists)}^{\mathrm{p}, \mathrm{r}}$ sentence:

$$
\forall x \exists y(\exists z /\{x\})(C(x) \rightarrow((P(y, x) \wedge z=1) \vee(N(y, x) \wedge z=0))) .
$$

Since this specific form of SAT is known to be NP-complete under quantifierfree reductions $([6])$, we could give an argument based on standard tools to show that $\mathrm{IF}_{\mathrm{AR}(\exists)}^{\mathrm{p}, \mathrm{r}}$ captures NP. In principle, we could extend this argument to show that an "infinite" version of SAT is complete for ESO under quantifier-free reductions, and thus $\operatorname{IF}_{\mathrm{AR}(\exists)}^{\mathrm{p}, \mathrm{r}}$ captures ESO. However, we will prove this result in the next section with a more direct argument.

\section{Explicit definition of Henkin quantifiers by signalling}

In this section we show that the prenex action recall fragment $\operatorname{IF}_{\mathrm{AR}(\exists)}^{\mathrm{p}, \mathrm{r}}$ has the same expressive power as the full IF logic. In the proof of this result we exploit the fact that existential second-order logic is captured by Henkin quantifiers with two rows:

Theorem 1 ([14]). For any ESO sentence there is an equivalent sentence of the form

$$
\left(\begin{array}{l}
\forall x_{1} \ldots \forall x_{n} \exists u \\
\forall y_{1} \ldots \forall y_{n} \exists v
\end{array}\right) \psi
$$

where $\psi$ is a quantifier-free formula.

By this result, it suffices to prove that, for any $n$, any sentence that is obtained by applying the Henkin quantifier $\mathrm{H}_{2}^{n}$ to a quantifier-free formula, is expressible in $\mathrm{IF}_{\mathrm{AR}(\exists)}^{\mathrm{p}, \mathrm{r}}$. Since $\mathrm{IF}_{\mathrm{AR}(\exists)}^{\mathrm{p}, \mathrm{r}}$ is a fragment of $\mathrm{IF}$, and $\mathrm{IF}$ is expressively equivalent to 
ESO, it follows then that the expressive powers of all the three logics $\mathrm{IF}_{\mathrm{AR}(\exists)}^{\mathrm{p}, \mathrm{r}}, \mathrm{IF}$ and ESO coincide.

Thus, we consider a sentence starting with the Henkin quantifier $\mathrm{H}_{2}^{n}$; let

$$
\varphi:=\left(\begin{array}{l}
\forall x_{1} \ldots \forall x_{n} \exists u \\
\forall y_{1} \ldots \forall y_{n} \exists v
\end{array}\right) \psi\left(x_{1}, \ldots, x_{n}, u, y_{1}, \ldots, y_{n}, v\right),
$$

where $\psi$ is a quantifier-free formula. In order to make the argument below more transparent, we formulate the truth condition of $\varphi$ in a slightly non-standard way: $M=\varphi$ if and only if there are relations $F_{a}, F_{b} \subseteq M^{n+1}$ such that

(a) $\left(M, F_{a}\right) \models \forall \bar{z} \exists w F_{a}(\bar{z}, w)$,

(b) $\left(M, F_{b}\right) \models \forall \bar{z} \exists w F_{b}(\bar{z}, w)$,

(c) $\left(M, F_{a}, F_{b}\right) \models \forall \bar{x} \forall u \forall \bar{y} \forall v\left(\neg F_{a}(\bar{x}, u) \vee \neg F_{b}(\bar{y}, v) \vee \psi(\bar{x}, u, \bar{y}, v)\right)$.

Here, and in the sequel, $\bar{z}$ denotes a tuple $\left(z_{1}, \ldots, z_{n}\right)$ of distinct variables; similarly, $\bar{x}=\left(x_{1}, \ldots, x_{n}\right)$ and $\bar{y}=\left(y_{1}, \ldots, y_{n}\right)$.

We will now build a sentence $\theta$ of $\mathrm{IF}_{\mathrm{AR}(\exists)}^{\mathrm{p}, \mathrm{r}}$ that expresses the three conditions above. The idea is to use the variables $\bar{z}$ and $w$ for expressing conditions (a) and (b), and the variables $\bar{x}, u, \bar{y}$ and $v$ for expressing (c). In addition we use an "index variable" $i$ that Abelard will use in the game $G(\theta, M)$ to separate the conditions (a), (b) and (c) from each other, and another "index variable" $j$ that Eloise uses either to signal the value of $i$, or to choose a disjunct of the quantifier-free part in (c).

To simplify the presentation, we assume first that the signature contains three constants, $\mathbf{a}, \mathbf{b}$ and $\mathbf{c}$, and consider only structures in which they are interpreted by distinct elements. In this case, the sentence $\theta$ is defined as follows:

$$
\theta:=\forall \bar{x} \forall u \forall \bar{y} \forall v \forall \bar{z} \forall i(\exists j / Z)(\exists w / X) \eta
$$

where $Z$ is the set $\left\{z_{1}, \ldots, z_{n}\right\}, X$ is the set $\left\{x_{1}, \ldots, x_{n}, u, y_{1}, \ldots, y_{n}, v, i\right\}$ and $\eta$ is the following quantifier-free formula

$$
\begin{aligned}
& (i=\mathbf{a} \rightarrow j=\mathbf{a}) \\
\wedge & (i=\mathbf{b} \rightarrow j=\mathbf{b}) \\
\wedge & (i=\mathbf{c} \wedge \bar{z}=\bar{x} \wedge j=\mathbf{a} \rightarrow w \neq u) \\
\wedge & (i=\mathbf{c} \wedge \bar{z}=\bar{y} \wedge j=\mathbf{b} \rightarrow w \neq v) \\
\wedge & (i=\mathbf{c} \wedge j=\mathbf{c} \rightarrow \psi(\bar{x}, u, \bar{y}, v)) \\
\wedge & (i=\mathbf{c} \rightarrow(j=\mathbf{a} \vee j=\mathbf{b} \vee j=\mathbf{c}))
\end{aligned}
$$

Lemma 1. Let $M$ be a structure such that $a \neq b \neq c \neq a$, where $a=\mathbf{a}^{M}$, $b=\mathbf{b}^{M}$ and $c=\mathbf{c}^{M}$. Then $M=\varphi$ if and only if $M=\theta$.

Proof. Assume first that $M \models \varphi$. Let $F_{a}$ and $F_{b}$ be relations satisfying the conditions (a), (b) and (c). Without loss of generality, we can assume that $F_{a}$ and $F_{b}$ are actually functions. We describe a winning strategy $\sigma$ for Eloise in the semantic game $G(\theta, M)$. In the first six moves of the game, Abelard chooses interpretations for the variables $\bar{x}, u, \bar{y}, v, \bar{z}$ and $i$; let $s$ be the assignment formed during these moves. Then Eloise answers by choosing a value $d_{s}$ for the variable $j$ as follows: 
- If $s(i)=a$, then Eloise sets $d_{s}=a$,

- If $s(i)=b$, then Eloise sets $d_{s}=b$,

- Assume then that $s(i)=c$. By condition (c), one of the following holds:

(i) $s(u) \neq F_{a}(s(\bar{x})), \quad$ (ii) $s(v) \neq F_{b}(s(\bar{y}))$, or $\quad$ (iii) $M, s \models \psi$.

In case (i), Eloise sets $d_{s}=a$; in case (ii), Eloise sets $d_{s}=b$; in case (iii),

Eloise sets $d_{s}=c$.

In the next move, Eloise chooses a value $e_{s}$ for the variable $w$. If $d_{s} \in\{a, b\}$, she sets $e_{s}=F_{d_{s}}(s(\bar{z}))$; in case $d_{s}=c$, she chooses an arbitrary $e_{s} \in M$.

Note that the choice of $d_{s}$ above does not depend on $s\left(z_{l}\right)$ for any $l$. Similarly, the choice of $e_{s}$ is independent of $s\left(x_{1}\right), \ldots, s\left(x_{n}\right), s(u), s\left(y_{1}\right), \ldots, s\left(y_{n}\right), s(v)$ and $s(i)$. Thus, the strategy of Eloise described above is uniform. Furthermore, it is straightforward to verify that Eloise has a winning strategy in $G\left(\eta, M, s^{\prime}\right)$, where $s^{\prime}=s\left(d_{s} / j, e_{s} / w\right)$. Thus, we see that $M \models \theta$.

Assume then for the other direction that $M \models \theta$. Then, given any assignment $s \in \operatorname{As}(X \cup Z, M)$, Eloise can choose values $d_{s}$ and $e_{s}$ for the variables $j$ and $w$ such that $d_{s}$ does not depend on $s(\bar{z}), e_{s}$ does not depend on $s(\bar{x} u \bar{y} v i)$ (but may depend on $d_{s}$ ), and Eloise has a winning strategy in the game $G\left(\eta, M, s\left(d_{s} / j, e_{s} / w\right)\right)$.

We define now relations $F_{a}, F_{b} \subseteq M^{n+1}$ as follows:

$-F_{a}:=\left\{\left(s(\bar{z}), e_{s}\right) \mid s \in \operatorname{As}(X \cup Z, M), d_{s}=a\right\}$,

$-F_{b}:=\left\{\left(s(\bar{z}), e_{s}\right) \mid s \in \operatorname{As}(X \cup Z, M), d_{s}=b\right\}$.

It suffices to show that conditions (a), (b) and (c) hold for these relations. In order to prove (a), let $\bar{m}=\left(m_{1}, \ldots, m_{n}\right) \in M^{n}$, and consider an assignment $s \in \operatorname{As}(X \cup Z, M)$ such that $s(\bar{z})=\bar{m}$ and $s(i)=a$. Then, $d_{s}$ is necessarily $a$, since otherwise Eloise would lose the game $G\left(\eta, M, s\left(d_{s} / j, e_{s} / w\right)\right)$ if Abelard chooses the first conjunct $i=\mathbf{a} \rightarrow j=\mathbf{a}$ of $\eta$. Thus, by the definition above, $\left(\bar{m}, e_{s}\right) \in F_{a}$. Condition (b) is proved symmetrically by using conjunct (2) of $\eta$.

Note that since the choice of $e_{s}$ depends only on $s(\bar{z})$ and $d_{s}$, we can assume that the relations $F_{a}$ and $F_{b}$ are actually functions $M^{n} \rightarrow M$.

To prove (c), let $s_{0}$ be an assignment with domain $X \backslash\{i\}$. We need to show that $F_{a}\left(s_{0}(\bar{x})\right) \neq s_{0}(u), F_{b}\left(s_{0}(\bar{y})\right) \neq s_{0}(v)$ or $M, s_{0}=\psi$. Let $s$ be an extension of $s_{0}$ to domain $X \cup Z$ such that $s(i)=c$. Then necessarily $d_{s} \in\{a, b, c\}$, since otherwise Eloise would lose the game $G\left(\eta, M, s\left(d_{s} / j, e_{s} / w\right)\right)$ if Abelard chooses the last conjunct (6) of $\eta$.

Assume first that $d_{s}=a$. Since the choice of $d_{s}$ does not depend on $s(\bar{z})$, we have $d_{s^{\prime}}=d_{s}=a$, where $s^{\prime}=s(s(\bar{x}) / \bar{z})$. Then by the definition of $F_{a}$, we have $e_{s^{\prime}}=F_{a}\left(s^{\prime}(\bar{z})\right)=F_{a}\left(s_{0}(\bar{x})\right)$. On the other hand, it must be the case that $e_{s^{\prime}} \neq s^{\prime}(u)=s_{0}(u)$, since otherwise Eloise would lose the game $G\left(\eta, M, s^{\prime}\left(d_{s^{\prime}} / j, e_{s^{\prime}} / w\right)\right)$ if Abelard chooses conjunct (3) of $\eta$. Thus, we see that $F_{a}\left(s_{0}(\bar{x})\right) \neq s_{0}(u)$. In the case $d_{s}=b$, we can prove in the same way that $F_{b}\left(s_{0}(\bar{y})\right) \neq s_{0}(v)$, by using conjunct (4) of $\eta$.

Assume finally, that $d_{s}=c$. Then it follows immediately that $M, s_{0} \models \psi$. This is because otherwise Eloise would lose the game $G\left(\eta, M, s\left(d_{s} / j, e_{s} / w\right)\right)$ if Abelard chooses conjunct (5) of $\eta$. 
We will next eliminate the assumption of three constants with distinct interpretations. On structures with at least two different elements, this is done by replacing the quantifiers $\forall i$ and $(\exists j / Z)$ in $\theta$ by the sequences $\forall i \forall i^{\prime} \forall i^{\prime \prime}$ and $(\exists j / Z)\left(\exists j^{\prime} / Z\right)\left(\exists j^{\prime \prime} / Z\right)$, respectively. Furthermore, the subformulae $i=\mathbf{a}, i=\mathbf{b}$ and $i=\mathbf{c}$ of $\eta$ are replaced by $i=i^{\prime} \wedge i \neq i^{\prime \prime}, i=i^{\prime \prime} \wedge i \neq i^{\prime}$ and $i^{\prime}=i^{\prime \prime} \wedge i \neq i^{\prime}$, and similarly for the subformulae $j=\mathbf{a}, j=\mathbf{b}$ and $j=\mathbf{c}$. Let $\theta^{\prime}$ be the formula obtained from $\theta$ by performing these changes. By a straightforward modification of the proof of Lemma 1, we see that $M=\varphi \Leftrightarrow M \models \theta^{\prime}$ holds for all structures $M$ with at least two elements.

If $M$ has only one element, then clearly $M \models \varphi \Leftrightarrow M \models \forall \bar{x} \forall u \forall \bar{y} \forall v \psi$. Furthermore, the implication $M \models \forall \bar{x} \forall u \forall \bar{y} \forall v \psi \Rightarrow M \models \varphi$ holds for all structures. Thus, we see that $\varphi$ is equivalent to $\theta^{*}$ on all structures, where $\theta^{*}$ is obtained from $\theta^{\prime}$ by adding (in the end of the prefix) the sequence $\forall \bar{x}^{\prime} \forall u^{\prime} \forall \bar{y}^{\prime} \forall v^{\prime}$ of universal quantifiers and the disjunct $\psi\left(\bar{x}^{\prime}, u^{\prime}, \bar{y}^{\prime}, v^{\prime}\right)$ to the quantifier-free part, for some fresh variables $\bar{x}^{\prime}=\left(x_{1}^{\prime}, \ldots, x_{n}^{\prime}\right), \bar{y}^{\prime}=\left(y_{1}^{\prime}, \ldots, y_{n}^{\prime}\right), u^{\prime}$ and $v^{\prime}$. This completes the proof of the main result in this section:

Theorem 2. $\operatorname{IF}_{\mathrm{AR}(\exists)}^{\mathrm{p}, \mathrm{r}}$ has the same expressive power as ESO. In particular, any class definable in $\mathrm{IF}$ is already definable in $\mathrm{IF}_{\mathrm{AR}(\exists)}^{\mathrm{p}, \mathrm{r}}$.

Note that the length of the $\mathrm{IF}_{\mathrm{AR}(\exists)}^{\mathrm{r}}$ translation $\theta^{*}$ given in the proof of Theorem 2 is only linear with respect to the length of the original $\mathrm{H}_{2}^{n}$ formula $\varphi$. Another interesting observation that follows from the proof is that there is no hierarchy of expressive power based on the number or length of signalling patterns: the signalling pattern in $\theta^{*}$ is independent of $n$.

\section{No-Henkin, no-signalling irregular IF logic}

In Section 4, we showed that all ESO properties can be defined by regular, prenex IF sentences of action recall. Such sentences are of the signalling, but not of the Henkin kind. We show now that, if the restrictions of regularity and prenex form are abandoned, then all ESO properties can be expressed by IF sentences which contain neither Henkin nor signalling patterns.

\section{Eliminating Henkin and signalling sequences by requantification}

In order to create a Henkin or a signalling pattern, we need to have two existential quantifiers that have certain dependencies to each other. In order to eliminate these patterns, we now attempt to replace existential quantifiers with universal quantifiers that have essentially the same role in the semantic game. This can be done by a simple trick if we allow requantification of variables.

Let $\varphi$ be a regular IF formula and let $y$ be a fresh variable. Suppose that $\varphi$ has a subformula of the form $(\exists x / V) \psi$. Now $(\exists x / V) \psi$ is equivalent to the irregular IF formula $(\exists y / V) \forall x(x \neq y \vee \forall y \psi)$. The truth of this equivalence can be seen by the following game-theoretical reasoning: After the quantification for $(\exists y / V)$, Abelard has to choose the same value for $x$ as Eloise chose for $y$, since 
else he loses the game (when Eloise chooses the left disjunct). Hence we see that Eloise can indirectly "force" Abelard to choose a value for $x$ in any ( $V$-uniform) way she wishes. And since Abelard may then choose a new value for $y$, Eloise cannot use its value for signalling later in the game.

By replacing $(\exists x / V) \psi$ with $(\exists y / V) \forall x(x \neq y \vee \forall y \psi)$ in the sentence $\varphi$, we obtain an equivalent sentence $\varphi^{*}$. If the existential quantifier $(\exists x / V)$ created Henkin or signalling patterns in $\varphi$ with some other quantifiers, these patterns are broken, since $x$ is not existentially quantified anymore in $\varphi^{*}$. The new existential quantifier $(\exists y / V)$, that was introduced, cannot create any new Henkin patterns since the fresh variable $y$ is not in any slash set of $\varphi^{*}$ and $(\exists y / V)$ has no existential quantifiers in its effective scope.

By iterating this translation process to every existential quantifier in $\varphi$, we obtain an equivalent formula $\varphi^{\prime}$ which has no Henkin patterns. But $\varphi^{\prime}$ has no signalling patterns either, since no existential quantifier in $\varphi^{\prime}$ is in the effective scope of any other existential quantifier.

Theorem 3. Every IF sentence $\varphi$ is equivalent to an irregular IF sentence $\varphi^{\prime}$ that has neither Henkin nor signalling patterns.

Proof. Let $\varphi$ be any IF-sentence. Let $\left\{x_{1}, \ldots, x_{k}\right\}$ be the set of (distinct) variables that are existentially quantified in $\varphi$. Let $\left\{y_{1}, \ldots, y_{k}\right\}$ be a set of distinct variables that do not occur in $\varphi$. We define $\varphi^{\prime}$ recursively as follows:

$$
\begin{aligned}
\varphi^{\prime} & =\varphi, \quad \text { if } \varphi \text { is a literal } \\
(\psi \vee \theta)^{\prime} & =\psi^{\prime} \vee \theta^{\prime},(\psi \wedge \theta)^{\prime}=\psi^{\prime} \wedge \theta^{\prime} \\
((\forall x / V) \psi)^{\prime} & =(\forall x / V) \psi^{\prime} \\
\left(\left(\exists x_{i} / V\right) \psi\right)^{\prime} & =\left(\exists y_{i} / V\right) \forall x_{i}\left(x_{i} \neq y_{i} \vee \forall y_{i} \psi^{\prime}\right) .
\end{aligned}
$$

By the observations that we did above, $\varphi^{\prime}$ has neither Henkin nor signalling patterns. Thus it suffices to show that $M \models \varphi$ if and only if $M \models \varphi^{\prime}$. This is quite easy to see by the game-theoretical intuition that we gave above. We give a formal proof for this equivalence in the appendix.

Note that our translation above can be applied for any IF formula - including non-prenex and irregular formulae. Furthermore, the translation increases the length of a given formula only by a small constant for each existential quantifier in it. If a sentence $\varphi$ in a prenex form is translated to $\varphi^{\prime}$ as above, the prenex form is lost. However, $\varphi^{\prime}$ is still "almost prenex" since only disjunctions with a literal as the left disjunct are created within the quantifier prefix. See the following example for explicitly expressing Henkin prefix $\mathrm{H}_{2}^{1}$ without using Henkin or signalling patterns.

Example 4. Consider the IF sentence $\forall x \exists y \forall z(\exists w /\{x, y\}) \psi$, where $\psi$ is quantifier free, and suppose that $y^{\prime}$ does not occur in $\psi$. By applying our translation procedure to the most external occurrence of an existential quantifier, $\exists y$, we obtain the formula $\xi:=\forall x \exists y^{\prime} \forall y\left(y \neq y^{\prime} \vee \forall y^{\prime} \forall z(\exists w /\{x, y\}) \psi\right)$. Note here that there is no need to apply the translation procedure to the innermost existential quantifier $(\exists w /\{x, y\})$. What happens to the flow of information in $\xi$ ? In the 
right disjunct, the variables $y$ and $y^{\prime}$ carry the same value; as a signal, $y$ is blocked by the slash set of $\exists w$ and, as a signal, $y^{\prime}$ is blocked by $\forall y^{\prime}$; but the value of $y$ (equal to the value of $y^{\prime}$ ) can still be used within $\psi$.

\section{Action recall for irregular formulae}

As we discussed after defining the restriction (RS), there are irregular formulae which satisfy it, but for which Eloise does not have action recall in the corresponding semantic game. We analyse here what would be the proper characterization of the syntactical fragment of action recall for irregular formulae.

Let $\varphi$ be an irregular IF sentence in which a variable $x$ is quantified two times. If $x$ is first universally quantified, this requantification does not violate action recall for Eloise, since the first value for $x$ is chosen by Abelard. But if $x$ is first existentially quantified, then there is a play of the semantic game for $\varphi$ in which Eloise chooses the value for $x$ and then forgets that value when $x$ is requantified, and thus she does not have action recall, supposing that Eloise has as at least one action make in the game after $x$ has been requantified.

By the observation above, the action recall fragment for Eloise for all (including irregular) formulae, denoted by $\mathrm{IF}_{\mathrm{AR}(\exists)}$, is obtained simply by requiring the following condition in addition to (RS):

- No quantifier $(Q x / V)$ is in the scope of a quantifier $(\exists x / W)$ such that a formula of the form $\psi \vee \theta$ or $(\exists y / U) \psi$ is in the scope of $(Q x / V)$.

\section{Conclusions}

We have shown that full IF (i.e., ESO) expressive power can be achieved, without the use of Henkin prefixes, already within each of the two following fragments of IF logic: 1) prenex, regular IF logic with action recall $\left(\operatorname{IF}_{\mathrm{AR}(\exists)}^{\mathrm{r}}\right)$, and 2) nonprenex, irregular IF logic without Henkin and signalling patterns. The proof of the first result shows that the $\mathrm{H}_{2}^{n}$ Henkin prefixes are explicitly definable by means of signalling prefixes with a constant number of signalling variables. Consequently, there are no hierarchies based on the number or length of signalling patterns in $\mathrm{IF}_{\mathrm{AR}(\exists)}^{\mathrm{r}}$.

These results extend the analysis of the expressive resources of IF logic which was initiated in [17] and [2], and they raise a number of questions to be further investigated:

- Is it possible to capture ESO within $\mathrm{IF}_{\mathrm{AR}(\exists)}^{\mathrm{r}}$ without the use of signalling? Note that this is not possible for $\mathrm{IF}_{\mathrm{AR}(\exists)}^{\mathrm{p}, \mathrm{r}}$, since prenex, regular IF logic without Henkin and signalling patterns collapses to first-order logic ([17]).

- When considering irregular prenex sentences, are there other sources of second-order expressive power, besides Henkin and signalling patterns?

- Are there interesting hierarchies of signalling prefixes, e.g. based on the number of universal or existential quantifiers? 


\section{Appendix}

\section{Example 1, and the prefix $\exists f \forall x$}

By applying so-called Skolemization, we can translate any IF sentence $\varphi$ to an equivalent ESO sentence of the form $\exists f_{1} \ldots \exists f_{n} \psi$, where $\psi$ is a first order formula that contains no existential quantifiers. (The functions $f_{i}$ here correspond to Eloise's "choice functions" for choosing values for the existentially quantified variables in $\varphi$.) For more details on Skolemization, see [15].

By applying Skolemization to $\forall x \exists y(\exists z / x)(U(x) \rightarrow(K(y) \wedge E(x, z)))$, we obtain an equivalent ESO sentence $\varphi:=\exists h \exists g \psi$, where

$$
\psi:=\forall x(U(x) \rightarrow(K(h(x)) \wedge E(x, g(h(x))))) .
$$

A proof that this ESO sentence defines the problem EXACT COVER BY 3SETS on appropriate structures can be found in [17]. Instead, we prove here that, if we restrict the class of adequate structures for the problem EXACT COVER BY 3-SETS by the additional constraint $K^{M} \subseteq M \backslash U^{M}$, then $\varphi$ captures the same class of structures as

$$
\varphi^{*}:=\exists f \forall x(U(x) \rightarrow(K(f(x)) \wedge E(x, f(f(x))))) .
$$

This, together with the arguments in [17], gives a new proof that the NPcomplete problem EXACT COVER BY 3-SETS is expressible by $\varphi^{*}$, that is, by the quantifier prefix $\exists f \forall x$. (Note that the additional constraint $K^{M} \subseteq M \backslash U^{M}$ does not decrease the generality of the problem.)

In one direction, it is apparent that $\varphi^{*}$ logically implies $\varphi$. Suppose instead that $\varphi$ holds in an appropriate structure $M$. Let $g, h: M \rightarrow M$ be two functions that satisfy $\psi$. Define

$$
f(x)=\left\{\begin{array}{l}
h(x) \text { if } x \in U^{M} \\
g(x) \text { if } x \in M \backslash U^{M}
\end{array}\right.
$$

Then, for all $a \in U^{M}$, we have $f(a)=h(a)$ and so from $h(a) \in K^{M}$ we obtain $f(a) \in K^{M}$; from our assumption that $K^{M} \subseteq M \backslash U^{M}$ we get $f(a) \in M \backslash U^{M}$; so, $g(h(a))=g(f(a))=f(f(a))$; then, from the fact that $(a, g(h(a))) \in E^{M}$ we deduce $(a, f(f(a))) \in E^{M}$. Therefore $M \models \varphi^{*}$.

\section{Example 2, DOMINATING SET}

We need to prove that the DOMINATING SET problem is captured by the sentence $\forall x \exists z(\exists y /\{x\})((E(y, x) \vee y=x) \wedge P(z))$. By using Skolemization, it suffices to prove this claim for the ESO sentence

$$
\zeta:=\exists f \exists g \forall x((E(g(f(x)), x) \vee g(f(x))=x) \wedge P(f(x))) .
$$

Fix an integer $k$. Let $G=\left(V, E^{G}, P^{G}\right)$ be any structure such that $\left(V, E^{G}\right)$ is a graph, and such that $P^{G}=\left\{d_{1}, \ldots, d_{k}\right\}$ is a subset of $V$ of cardinality $k$. Suppose first that $G$ has a dominating set $D$ of cardinality $k$. Enumerate $D$ as $\left\{c_{1}, \ldots, c_{k}\right\}$. Since $D$ is a dominating set, to each $a \in V$ we can associate a $b_{a} \in D$ such that either $\left(b_{a}, a\right) \in E^{G}$ or $b_{a}=a$. Now, define $f: V \rightarrow P^{G}$ as follows: if $b_{a}=c_{i}$, then set $f(a):=d_{i}$. Define $g: V \rightarrow V$ as follows: $g\left(d_{i}\right)=c_{i}$; 
for $a \in V \backslash P^{G}, g(a)$ takes an arbitrary value. Note then that, by the definitions, for every $a \in V, g(f(a))=b_{a}$. Therefore, $(G, f, g) \models E(g(f(x)), x) \vee g(f(x))=x$. And the definition of $f$ implies that $(G, f, g) \models P(f(x))$.

Suppose instead that $G \models \zeta$. Then, there are functions $f: V \rightarrow P^{G}$ and $g: V \rightarrow V$ such that, for every $a \in V$, either $(g(f(a)), a) \in E^{G}$ or $g(f(a))=a$. Define $D:=g\left[P^{G}\right]=\left\{g(a) \mid a \in P^{G}\right\}$. Clearly $\operatorname{Card}(D) \leq \operatorname{Card}\left(P^{G}\right)=k$, and since $g(f(a)) \in D$ for every $a \in V, D$ is a dominating set.

\section{Example 3, SAT by signalling}

For proving that $\forall x \exists y(\exists z /\{x\})(C(x) \rightarrow((P(y, x) \wedge z=1) \vee(N(y, x) \wedge z=0)))$ captures SAT, we apply Skolemization again and prove the claim for the ESO sentence $\xi:=\exists f \exists g \psi$, where

$$
\psi:=\forall x(C(x) \rightarrow((P(f(x), x) \wedge g(f(x))=1) \vee(N(f(x), x) \wedge g(f(x))=0))) .
$$

Let $M$ be an appropriate structure, and $\pi$ the propositional formula encoded by it. Suppose first that $M$ is a "yes" instance of SAT; then there is a truth assignment $T$ such that each clause $c$ of $\pi$ contains a literal $\alpha_{c}$ for which we have $T\left(\alpha_{c}\right)=1$. A literal $\alpha_{c}$ can either be of the form $p_{c}$ or $\neg p_{c}$, with $p_{c}$ a proposition symbol. In the first case, we then have $T\left(p_{c}\right)=1$, while in the second $T\left(p_{c}\right)=0$. Let $f: M \rightarrow M$ be the function that maps $c$ to $p_{c}$ (define it arbitrarily on elements that are not clauses); let $g: M \rightarrow M$ be defined by $g(p):=T(p)$ if $p$ is a proposition symbol, and an arbitrary constant otherwise. With these $f$ and $g$, $(M, f, g) \models \psi$.

Vice versa, suppose $M \models \xi$. Let $f, g$ be two functions that satisfy $\psi$. Let $T$ be a truth assignment such that $T(p)=g(p)$ for all the proposition symbols in $\pi$. Now for any $c \in C^{M}$, either $(f(c), c) \in P^{M}$ and $g(f(c))=1$, or $(f(c), c) \in N^{M}$ and $g(f(c))=0$. In the former case $f(c)$ is a proposition symbol occurring positively in $c$, to which $T$ assigns truth value 1 . Similarly, in the second case $f(c)$ is a proposition symbol which occurs negatively in $c$, to which $T$ assigns truth value 0 . These remarks show that $T$ satisfies $\pi$.

\section{Theorem 3; a proof for the equivalence}

We argue by using compositional so-called team semantics for IF logic. For the definition of this semantics - and the notation and terminology that we use here - refer to [15]. In the proof we also use the downwards closure property of IF logic. That is, if $M, X \models \varphi$ and $Y \subseteq X$, then $M, Y \models \varphi$.

Let $\mu$ be a subformula of $\varphi$ and let $X$ be a team for which $\operatorname{dom}(X)=\operatorname{Free}(\mu)$. We show by the structural induction on $\varphi$ that the following equivalence holds for any team $Y$ for which $\operatorname{dom}(Y)=\left\{y_{1}, \ldots, y_{k}\right\}$ :

$$
M, X \models \mu \quad \text { iff } \quad M, X \times Y=\mu^{\prime},
$$

where $X \times Y:=\left\{s \cup s^{\prime} \mid s \in X\right.$ and $\left.s^{\prime} \in Y\right\}$. (Note that $\operatorname{dom}(X) \cap \operatorname{dom}(Y)=\emptyset$.)

- The case when $\mu$ is a literal holds trivially since then $\mu=\mu^{\prime}$ and the values of the variables $y_{i}$ in the team do not affect the truth of $\mu$. 
- The cases $\mu=\psi \wedge \theta$ and $\mu=\left(\forall x_{j} / V\right) \psi$ are proven easily by just applying the inductive hypothesis.

- Let $\mu=\psi \vee \theta$. Suppose first that $M, X \models \psi \vee \theta$ and let $Y$ be team. Now there are $X_{1}, X_{2} \subseteq X$ s.t. $X_{1} \cup X_{2}=X, M, X_{1} \models \psi$ and $M, X_{2} \models \theta$. By the inductive hypothesis we have $M, X_{1} \times Y=\psi^{\prime}$ and $M, X_{2} \times Y=\theta^{\prime}$. Since $X_{1} \times Y \cup X_{2} \times Y=X \times Y$, we have $M, X \times Y=\psi^{\prime} \vee \theta^{\prime}$, i.e. $M, X \times Y \models \mu^{\prime}$. Suppose then that $M, X \times Y \models \mu^{\prime}$ for any $Y$. In particular $M, X \times\{r\} \models \mu^{\prime}$ for some singleton $\{r\}$. Now there are $X_{1}, X_{2} \subseteq X$ s.t. $X_{1} \cup X_{2}=X$, such that $M, X_{1} \times\{r\} \models \psi^{\prime}$ and $M, X_{1} \times\{r\} \models \theta^{\prime}$. By the inductive hypothesis we have $M, X_{1} \models \psi$ and $M, X_{2} \models \theta$. Therefore $M, X \models \psi \vee \theta$, i.e. $M, X \models \mu$.

- Let $\mu=\left(\exists x_{j} / V\right) \psi$ for some $j \leq k$. Suppose first that $M, X \models \varphi$ and let $Y$ be a team. Hence there is a $V$-uniform $f: X \rightarrow M$ s.t. $M, X\left[f / x_{j}\right] \models \psi$.

Let $g: X \times Y \rightarrow M$ s.t. $g(s)=f(s \uparrow \operatorname{dom}(X))$ for every $s \in X \times Y$. We define the teams $X^{\prime}:=(X \times Y)\left[g / y_{j}, M / x_{j}\right], X_{1}:=\left\{s \in X^{\prime} \mid x_{j} \neq y_{j}\right\}$ and $X_{2}:=\left\{s \in X^{\prime} \mid x_{j}=y_{j}\right\}$. Now clearly $X_{1} \cup X_{2}=X^{\prime}$ and $M, X_{1} \models x_{j} \neq y_{j}$. Since $M, X_{2} \models x_{j}=y_{j}$, by the definition of $g$ it is quite easy to see that

$$
X_{2}\left[M / y_{j}\right]=\left(X\left[f / x_{j}\right]\right) \times\left(Y\left[M / y_{j}\right]\right)
$$

By the inductive hypothesis we have $M,\left(X\left[f / x_{j}\right]\right) \times\left(Y\left[M / y_{j}\right]\right) \models \psi^{\prime}$ and therefore $M, X_{2}\left[M / y_{j}\right] \models \psi^{\prime}$. Furthermore we have $M, X_{2} \models \forall y_{j} \psi^{\prime}$ and thus $M, X^{\prime} \models x_{j} \neq y_{j} \vee \forall y_{j} \psi^{\prime}$. Since $f$ was $V$-uniform also $g$ is $V$-uniform and thus $M, X \times Y \models\left(\exists y_{j} / V\right) \forall x_{j}\left(x_{j} \neq y_{j} \vee \forall y_{j} \psi^{\prime}\right)$, i.e. $M, X \times Y \models \mu^{\prime}$.

Suppose then that $M, X \times Y \models \mu^{\prime}$ for any $Y$. In particular $M, X \times\{r\} \models \mu^{\prime}$ for some singleton $\{r\}$. Now there is a $V$-uniform function $g: X \times\{r\} \rightarrow M$ s.t. $M, X^{\prime} \models x_{j} \neq y_{j} \vee \forall y_{j} \psi^{\prime}$, where $X^{\prime}=(X \times\{r\})\left[g / y_{j}, M / x_{j}\right]$. Hence there are $X_{1}, X_{2} \subseteq X^{\prime}$ such that $M, X_{1} \models x_{j} \neq y_{j}$ and $M, X_{2} \models \forall y_{j} \psi^{\prime}$. Let $Z:=\left\{s \in X^{\prime} \mid s\left(x_{j}\right)=s\left(y_{j}\right)\right\}$. Since $M, Y_{1} \models x_{j} \neq y_{j}$ we must have $Z \subseteq X_{2}$. Thus by downwards closure $M, Z \models \forall y_{j} \psi^{\prime}$, i.e. $M, Z\left[M / y_{j}\right] \models \psi^{\prime}$.

Let $f: X \rightarrow M$ be such that $f(s)=g(s \cup r)$. Since $M, Z \models x_{j}=y_{j}$, by the definition of $f$ it is quite easy to see that

$$
\left(X\left[f / x_{j}\right]\right) \times\{r\} \subseteq Z\left[M / y_{j}\right] .
$$

Therefore, again by downwards closure, $M,\left(X\left[f / x_{j}\right]\right) \times\{r\} \models \psi^{\prime}$. By the inductive hypothesis $M, X\left[f / x_{j}\right] \models \psi$. Since $g$ was $V$-uniform also $f$ is $V$ uniform and thus we have $M, X \models\left(\exists x_{j} / V\right) \psi$, i.e. $M, X \models \mu$.

By Theorem 5.2 of [3] the values of variables in a team cannot affect the truth of an IF sentence. Therefore, when $\mu=\varphi$ and $X=\{\emptyset\}$, the equivalence in the proof of Theorem 3 follows from $(\star)$. This concludes the proof.

\section{References}

1. F. Barbero, On existential declarations of independence in IF logic, The Review of Symbolic Logic 6 (2013) 254-280. 
2. F. Barbero, Complexity of syntactical tree fragments of Independence-Friendly logic, pre-print, arXiv:1610.03406.

3. X. Caicedo, F. Dechesne, T. M. V. Janssen, Equivalence and quantifier rules for logic with imperfect information, Logic Journal of the IGPL 17 (2009) 91-129.

4. X. Caicedo, M. Krynicki, Quantifiers for reasoning with imperfect information and $\Sigma_{1}^{1}$-logic, in: I. M. L. O. W. A. Carnielli and (ed.), Contemporary Mathematics, vol. 235, American Mathematical Society, 1999, pp. 17-31.

5. P. Cameron, W. Hodges, Some combinatorics of imperfect information, Journal of Symbolic Logic 66 (2001) 673-684.

6. E. Dahlhaus, Reduction to NP-complete problems by interpretations, Proceedings of the Symposium "Rekursive Kombinatorik" on Logic and Machines: Decision Problems and Complexity (1983) 357-365.

7. H. B. Enderton, Finite partially ordered quantifiers, Mathematical Logic Quarterly 16 (8) (1970) 393-397.

8. E. Grandjean, First-order spectra with one variable, Journal of Computer and System Sciences 40 (1990) 136-153.

9. L. Henkin, Some remarks on infinitely long formulas, in: Infinitistic methods, Pergamon Press, Oxford-London-New York-Paris, 1961.

10. J. Hintikka, G. Sandu, Informational independence as a semantical phenomenon, in: J. E. Fenstad et al (ed.), Logic, Methodology and Philosophy of Science VIII, Elsevier Science Publishers B.V., 1989, pp. 571-589.

11. W. Hodges, Compositional semantics for a language of imperfect information, Logic Journal of the IGPL 5 (1997) 539-563.

12. W. Hodges, Some strange quantifiers, in: J. Mycielski, G. Rozenberg, A. Salomaa (eds.), Structures in Logic and Computer Science. Lecture Notes in Computer Sci. vol.1261, Springer-Verlag London, UK, 1997, pp. 51-65.

13. T. Hyttinen, T. Tulenheimo, Decidability of IF modal logic of perfect recall, in: Advances in Modal Logic, vol. 5, 2005.

14. M. Krynicki, Hierarchies of partially ordered connectives and quantifiers, Math. Log. Q. 39 (1993) 287-294. URL http://dx.doi.org/10.1002/malq.19930390134

15. A. L. Mann, G. Sandu, M. Sevenster, Independence-Friendly Logic - a GameTheoretic Approach, vol. 386 of London Mathematical Society lecture note series, Cambridge University Press, 2011.

16. M. Sevenster, Branches of imperfect information: logic, games, and computation, Ph.D. thesis, ILLC, Universiteit van Amsterdam (2006).

17. M. Sevenster, Dichotomy result for independence-friendly prefixes of generalized quantifiers, The Journal of Symbolic Logic 79(04) (2014) 1224-1246.

18. J. Väänänen, Dependence Logic: A New Approach to Independence Friendly Logic, vol. 70 of London Mathematical Society Student Texts, Cambridge University Press, 2007.

19. J. Virtema, Approaches to finite variable dependence, Ph.D. thesis, University of Tampere (2014). 